\title{
Evolutionary history is a powerful means to evince significant levels of plant services for humankind
}

\author{
Rafael Molina-Venegas ( $\nabla$ rmolina@us.es ) \\ University of Alcalá https://orcid.org/0000-0001-5801-0736 \\ Miguel Rodriguez \\ University of Alacala \\ Manuel Pardo-de-Santayana \\ Universidad Autónoma de Madrid \\ Cristina Ronquillo \\ Universidad de Alcalá \\ David J. Mabberley \\ National Herbarium of New South Wales
}

\section{Brief Communication}

Keywords: Biodiversity, Plant-use Records, Vascular Plant Phylogeny, Human Benefits

Posted Date: December 11th, 2020

DOI: https://doi.org/10.21203/rs.3.rs-121288/v1

License: (c) (1) This work is licensed under a Creative Commons Attribution 4.0 International License.

Read Full License

Version of Record: A version of this preprint was published at Nature Ecology \& Evolution on March 29th, 2021. See the published version at https://doi.org/10.1038/s41559-021-01414-2. 


\title{
Evolutionary history is a powerful means to evince significant levels of plant services for humankind
}

\author{
Rafael Molina-Venegas ${ }^{1 *}$, Miguel Ángel Rodríguez ${ }^{1}$, Manuel Pardo-de-Santayana ${ }^{2,3}$, Cristina Ronquillo ${ }^{1}$ \\ \& David J. Mabberley ${ }^{4}$
}

1. GLOCEE - Global Change Ecology and Evolution Group, Department of Life Sciences, Universidad de Alcalá, 28805, Spain.

2. Department of Biology (Botany), Universidad Autónoma de Madrid, 28049, Spain.

3. Centro de Investigación en Biodiversidad y Cambio Global (CIBC-UAM)

4. Wadham College, University of Oxford, OX1 3PN, United Kingdom; Department of Biology, Macquarie University, and National Herbarium of New South Wales, Sydney, 2000, Australia.

*Correspondence to: rafmolven@gmail.com

The divergent nature of evolution suggests that securing the human benefits that are directly provided by biodiversity may require counting on disparate lineages of the Tree of Life. However, quantitative evidence connecting evolutionary history to human well-being is still surprisingly tenuous. Here, we drew on a global review of plant-use records and the most comprehensive vascular plant phylogeny available demonstrating that, at any sample size, maximum levels of phylogenetic diversity captured significantly greater numbers of plant-use records than random selection, both globally and across the main continental regions of the world. Our study establishes an empirical foundation that links evolutionary history to human well-being, and it will serve as a discussion baseline to promote better-grounded accounts of the services that are directly provided by biodiversity. 
Evolution is the process that led to all living organisms and hence the foundation for the human benefits that are directly provided by biodiversity ${ }^{1}$, including not only basic resources but also psychological and self-fulfilment needs ${ }^{2}$. Since evolution is a divergent process, some researchers have claimed that securing the services that are directly provided by biodiversity may require counting on disparate lineages of the Tree of Life ${ }^{1}$, because they might provide complementary benefits ${ }^{3}$. Although this theoretical background is deeply rooted in the academic literature $^{4-6}$, empirical evidence connecting evolutionary history to human well-being is still surprisingly tenuous ${ }^{7}$ and not without controversy ${ }^{8,9}$. While some authors hold that maximizing phylogenetic diversity should lead to recognition of high levels of useful feature diversity ${ }^{1,8}$, others have suggested that the phylogenetic approach can be misleading ${ }^{9}$. This controversy likely reflects that the connection between evolutionary history and human well-being remains largely theoretical ${ }^{10}$ (but see Forest et al. ${ }^{11}$ for an empirical local assessment), which is only an initial move towards its consolidation as a scientific paradigm.

Here, we provide quantitative evidence that maximum levels of global plant phylogenetic diversity $\left(\mathrm{PD}_{\max }\right)$ capture more human benefits (i.e. plant-use records sorted into 28 standard categories of use ${ }^{12}$ ) and at higher diversity levels (i.e. records more evenly distributed between the categories) than does random selection of taxa, supporting the longstanding notion that maximizing phylogenetic diversity is a valuable means to retrieve high levels of useful feature diversity ${ }^{4-6}$. Our genus-level analysis is based on the most comprehensive time-calibrated vascular plant phylogeny available ${ }^{13,14}$, including all accepted vascular plant genera worldwide (a total of 13489) as well as 9478 genus-level plant-use records (presence/absence) obtained from a systematic review of botanical literature and authoritative websites ${ }^{15}$.

The $\mathrm{PD}_{\max }$ strategy overcame random selection of taxa at any sample size (Fig. 1a), with relative gains varying between $4 \%$ and $46 \%$ (Fig. 1b). This result suggests that in the absence of any other source of information beyond evolutionary history, prospecting disparate lineages of the phylogeny could help to make the most of the natural services that are the result of evolution. With regard to individual plant-use categories, $\mathrm{PD}_{\max }$ retrieved a higher number of 
records relative to random selection in $92 \%$ of the comparisons (Fig. 2 and Supplementary Fig. $\mathrm{S} 1)$. Moreover, given that relative record gains with $\mathrm{PD}_{\max }$ were overall higher for the less common categories (Fig. S2), $\mathrm{PD}_{\max }$ also retrieved significantly more equitable distributions of records among categories at most sample sizes (Fig. 1c). This indicates that $\mathrm{PD}_{\max }$ recovers more plant-uses in general than random selection, and that it does so optimizing the capture of some of the rarest uses, thus resulting in a more balanced palette of human benefits. Both $\mathrm{PD}_{\max }$ and random selection strategies retrieved the maximum possible richness of plant-use categories $(\mathrm{n}=28)$ across most sample sizes, yet random selection failed in retrieving maximum richness of categories at $10 \%$ and $20 \%$ sample sizes in a few cases (data not shown). While our genuslevel approach is superior to the species level in that the latter would suffer from unacceptable omission errors -ethnobotanical knowledge will most likely remain vastly under-documented for long below the genus level ${ }^{16-18}$ - and extreme lack of phylogenetic information ${ }^{13}$, it may introduce some uncertainty because the operational unit of plant-use is often the species. As such, retrieving a useful genus that comprises just a few species could be considered more valuable than a highly diversified one with the same use, because the uncertainty regarding the species that are actually useful within each genus would be less in the former case. Nonetheless, a reanalysis of the data after downweighting our genus-level plant-use observations in direct proportion to species richness per genus revealed an even stronger pattern (Supplementary Fig. S3). Moreover, the relationship between PD and plant benefits held in separate continental regions of the world (TDWG level-1 standards, Supplementary Figs. S4-S5), which suggests that our results are consistent across floras that have evolved in distinct biogeographic regions and over different timescales.

The striking success of the $\mathrm{PD}_{\max }$ strategy lies in the phylogenetic structure of the categories. As such, we found a strong positive relationship between the PD that is encapsulated by each plant-use category and the relative gain in records per category under the $\mathrm{PD}_{\max }$ strategy (Fig. 3), meaning that greater gains are predicted for phylogenetically dispersed categories. In fact, the only category that was significantly underrepresented with $\mathrm{PD}_{\max }$ relative to random 
selection concerns rubber plants (Fig. 2 and Supplementary Fig. S1), which are strongly clumped in the phylogeny (Supplementary Table S2). Our results complement previous findings reported in local studies that high levels of PD can increase multifunctionality via complementarity of beneficial attributes among phylogenetically distant taxa ${ }^{3}$. For example, regarding the production of natural poisons against harmful or nuisance invertebrates, we found that maximum levels of global PD capture more plant taxa generating them than random selection (Fig. 2), which in turn may imply an increased potential to control the detrimental effects of disparate invertebrate lineages. While the latter hypothesis cannot be tested with our data, observations that most of the antagonistic plant-invertebrate interactions that ultimately shaped this benefit are phylogenetically conserved ${ }^{19,20}$ (i.e. invertebrate species often attack a narrow range of closely-related host plants) and geographically restricted ${ }^{21}$ support this idea. It follows that, in the shadow of global change, counting on a variety of invertebrate poisons and deterrents from distinct plant lineages may help to counter phylogenetically diverse pests coming from disparate parts of the world ${ }^{22,23}$.

It is important to note that an unobserved link between a human need and a taxon does not necessarily imply that the link will not be found in the future. The ecological apparency hypothesis states that among equally valuable taxa with regard to a certain use, the most apparent or salient ones are preferred simply because they are readily available ${ }^{24}$. Furthermore, cultural factors could also explain the preferential use of certain taxa at the expense of others that might equally fulfill the need ${ }^{25}$. By analogy to the ecological prediction that higher competition between closely-related taxa of similar phenotypes can lead to greater phylogenetic diversity $^{26}$, human preference patterns in the use of available plant resources might have increased phylogenetic overdispersion in local ethnofloras. Therefore, ecological and cultural factors, together with the fact that both plant lineages and the human cultures that prospect them are geographically restricted to a greater or lesser extent, may have contributed to the striking success of the $\mathrm{PD}_{\max }$ strategy over random selection in capturing the human benefits that are associated with plant biodiversity. 
Services (IPBES) has recently approved undertaking the assessment of the use of 'wild' species, including the identification of opportunities to establish measures that ensure and promote sustainable practices ${ }^{27}$. Concurring with the IPBES philosophy that the world is in need of a broadly appreciation of nature's contribution to people ${ }^{28,29}$, our study establishes a solid empirical foundation that links evolutionary history to human well-being, and it will serve as a discussion baseline to promote better-grounded accounts of the services that are directly provided by biodiversity ${ }^{30,31}$.

\section{Methods}

Plant-use dataset. We compiled a genus-level dataset of plant-use records for all vascular plant taxa described to date using the information gathered in the fourth edition of Mabberley's plantbook $^{15}$. Mabberley's plant-book is the most comprehensive and authoritative encyclopaedic review of global plant classification (genera) and their uses published hitherto. From 1974 to 2017 all the information included in Mabberley's plant-book was gathered, sorted, evaluated including modern Floras, handbooks, periodicals, monographs and websites (all references can be found in Mabberley ${ }^{15}$. We conducted a double-check manual screening of all plant-uses described in Mabberley's plant-book and sorted them into 28 standard categories of use following the guidelines in the Economic Botany Data Collection Standard ${ }^{12}$ (hereafter "Collection Standard"). When two or more applications of the same category were described for a given taxon, we considered them as a single plant-use record. For example, if the wood of a taxon is used to build poles, furniture and toys (i.e. three different applications), we simply recorded that the taxon provides timber. This procedure resulted in a binary classification of 9478 plant-use records across the 28 categories, including benefits related to human and animal nutrition (human food, human-food additives, vertebrate food, invertebrate food), materials (wood, stems, fibres, leaves, seeds/fruits, tannins/dyestuffs, gums/resins, lipids, waxes, scents, latex/rubber), fuels (fuelwood, charcoal, biofuels), medicine (both human and veterinary), 
poisons (vertebrate poison, invertebrate poison), social (antifertility agents, smoking materials/drugs, symbolic/magic/inspiration) and environmental uses (ornamental, bioindicators/bioremediators, soil improvers, hedging/shelter). A detailed description of the categories is provided in Supplementary Table S1. Despite the use of leaves and seeds/fruits as materials are considered as "miscellaneous" in the Collection Standard, we took them up front as independent categories because we found many records in Mabberley's plant-book that fit into these categories (typically leaves for thatching and seeds/fruits for handicrafts). The environmental categories "erosion control", "revegetators", "soil improvers" and "agroforestry" described in the Collection Standard were considered as one single category (i.e. soil improvers) because they were very difficult to tease apart in many cases (e.g. some plants are used in agroforestry because they prevent soil erosion, and revegetators often improve soil quality). The same rationale applies to the Collection Standard categories "shade/shelter" and "boundaries/barriers/supports", which were merged into a single category (i.e. hedges and shelters). The Collection Standard also recognized different sub-categories of medicine, human food and poisons ${ }^{12}$, but we did not distinguish between them here because such information is often unknown and does not make much sense in the context of our global assessment. For example, while we are interested in recording the value of a taxon as human food, distinguishing between the parts of the plant that are actually eaten (sub-categories for human food in the Collection Standard) is rather irrelevant for the purposes of the study. A few records could not be assigned to any of the categories described in the Collection Standard (e.g. spores and inflorescences used as materials), which recommends gathering such cases into "miscellaneous" categories ${ }^{12}$. However, we simply disregarded them because such a mixture of poorly represented categories would not make sense in the context of our study. Finally, the category "cork and cork substitutes" described in the Collection Standard was disregarded because we found very few records in Mabberley's plant-book (likely because cork and cork substitutes are provided only by a few species and primarily from Quercus). We considered both fully realized ( $>99 \%$ of the cases) and mooted uses (as long as they were properly documented in the literature), and doubtful entries were disregarded in any case. The resultant 
plant-use binary matrix (i.e. presence/absence of uses per genus) was used in all the analyses described below. Additionally, we derived a downweighted plant-use matrix by dividing the entries in the binary matrix (plant-use observations at the genus-level) by the total number of accepted species per genus (following Plants of the World Online ${ }^{32}$ ). This second matrix was used in a second round of analyses to take into account the uncertainty in the relationship between plant-use records in the genus-level dataset and the species that are actually useful, as the latter information is often unknown.

Of all the taxa included in the dataset, $33 \%$ showed at least one category of use, with a maximum number of plant-use records per taxa of 17 (Supplementary Fig. S6). The most common category was "ornamental" (26\%), followed by "medicine" (16\%), "human food" $(13 \%)$ and "timber" (8\%), while the rest of categories occurred at a frequency lower than $5 \%$ (Supplementary Fig. S7). The phi correlation coefficient among the categories varied between 0.008 and 0.332 , suggesting overall weak relationships among them.

Phylogenetic data. We generated a genus-level time-calibrated molecular phylogeny using the mega-tree GBOTB.extended ${ }^{14}$, which is a combination of the GBOTB tree for seed plants of Smith \& Brown ${ }^{13}$ and the pteridophytes clade in Zanne et al..$^{33}$ phylogeny with updates and corrections (i.e. taxonomic standardization to The Plant List ${ }^{34}$ nomenclatural and spelling criteria). This combined phylogeny represents the most comprehensive and sophisticated molecular phylogeny for vascular plants published hitherto. For each accepted genus in Mabberley's plant-book, we picked one representative species at random from the largest monophyletic cluster of the genus in GBOTB.extended (if available). In the very few cases where more than one largest monophyletic cluster was found, we first selected one of the clusters at random and then picked one representative species. The GBOTB.extended phylogeny was then pruned to retain only the representative species of the genera. After resolving a few discrepancies and synonymy issues between Mabberley's plant-book ${ }^{15}$ and The Plant List ${ }^{34}$ (using the nomenclatural criteria in Plants of the World Online $e^{32}$ as a complementary reference to solve disputes), we found that $71 \%$ of the genera accepted in Mabberley's plant-book 
included at least one representative species in the phylogeny. This purely molecular phylogenetic topology (hereafter "molecular tree") revealed that all the taxonomic families of the genera included in the tree formed monophyletic clades except for Nymphaeaceae, Olacaceae, and Tectariaceae, which were paraphyletic, and the polyphyletic Diplaziopsidaceae (see Supplementary Table S3 for a list of genera with taxonomic families). To take into account uncertainty in the phylogenetic relationships of the taxa that were missed in the molecular tree (hereafter "phylogenetically uncertain taxa" or $\mathrm{PUT}^{35}$ ), we derived a distribution of phylogenetic hypotheses from the latter using a systematic randomization procedure that was taxonomically and phylogenetically informed ${ }^{35}$. The workflow proposed by Rangel et al. ${ }^{35}$ implies defining for each PUT its "most derived consensus clade" (MDCC) (i.e. the clade in the molecular tree that most certainly contains the PUT) based on expert knowledge (e.g. taxonomy, morphology, geographic distribution, etc). Once the MDCCs of the PUTs are defined, a distribution of phylogenetic hypotheses can be generated by replicating the random insertion of the PUTs within their respective MDCCs a high number of times (e.g. 100 times per posterior tre $\mathrm{e}^{35}$ ). The resultant phylogenetic hypotheses can be then used to replicate the analyses and average the results over the entire distribution of trees ${ }^{9,14,35}$. Smith $\&$ Brown $^{13}$ provided just one maximum likelihood tree rather than a posterior distribution, and therefore we derived 100 alternative phylogenetic hypotheses from the maximum likelihood tree as follows.

First, we retrieved for each genus in the dataset the taxonomic rank immediately above in the taxonomic hierarchy (typically subtribe, tribe or subfamily in ascending order, hereafter "taxonomic ranks") from the NCBI Taxonomy database, the standard nomenclature and classification repository for the International Nucleotide Sequence Database Collaboration ${ }^{36}$. For some families, this information was not available in the NCBI repository, in which case we retrieved the taxonomic ranks from Mabberley's plant-book ${ }^{15}$. In the cases where taxonomic ranks were neither available in the latter source, we simply assigned the family rank to the genera. The mapping of taxonomic ranks in the molecular tree reveals whether or not they represent natural lineages (i.e. monophyletic or paraphyletic ${ }^{37}$ ), and we took advantage of such information to define the MDCCs for our PUTs. If the taxonomic rank of a PUT mapped as 
purely monophyletic or purely paraphyletic in the molecular tree, the subset of phylogenetic branches connecting all the genera in the tree that shared the same taxonomic rank as the PUT (hereafter "sharing taxa") defined the MDCC (see Supplementary Figs. S8a and S9a). In few cases, the taxonomic ranks did not map as purely monophyletic or paraphyletic due to (1) the presence of "outliers" that mapped away from the main cluster of sharing taxa or (2) the presence of "intruders" from a different taxonomic rank within the main cluster. Such outliers and intruders might represent incorrect taxonomic assignments or even artefacts derived from the phylogenetic inference rather than evidence of unnatural (i.e. polyphyletic) groups. Thus, we calculated two different indices for each potential monophyletic or paraphyletic cluster of sharing taxa (because of the presence of outliers, intruders, or both) in the phylogeny. The outlier ratio (OR) for a given set of sharing taxa is the ratio between the number of outliers observed for the set (relative to the largest cluster) and the number of sharing taxa in the set, and the intruder ratio (IR) is the ratio between the number of intruders observed within the largest cluster of sharing taxa and the size of the cluster (see Supplementary Figs. S8 and S9). If (and only if) both ratios were $\leq 0.05$, the subset of phylogenetic branches connecting all the sharing taxa in the largest cluster (i.e. including intruders if any but not outliers) defined the MDCC of the PUT. Otherwise, the MDCC was defined as the smallest phylogenetic clade that included all the sharing taxa in the tree (i.e. including outliers and/or intruders, see Supplementary Figs. S8 and S9). In those cases where one single genus represented the only sharing taxon of a PUT in the molecular tree, the terminal node (i.e. the phylogenetic tip) defined the MDCC of the PUT only if the node represented a singleton taxonomic family or subfamily. Otherwise (e.g. singleton tribes or subtribes), the parent node of the singleton sharing taxon defined the MDCC instead (see Supplementary Fig. S10). Once all the PUTs were assigned to a MDCC (see Supplementary Table S4), they were added to a randomly selected branch of their corresponding MDCC, the probability of being added along any branch of the clade being directly proportional to the length of the branch. We used a uniform distribution to determine the exact position to insert the PUTs along the selected branches ${ }^{38}$. This procedure was replicated 100 times to obtain a distribution of phylogenetic hypotheses. 
249 Finding the subsets of genera that maximize phylogenetic diversity. We used the phylogenetic diversity (PD) index as a metric of the evolutionary history encompassed by a set phylogenetic diversity ${ }^{4,8,9,39}$. The greedy algorithm ${ }^{40}$ was used to find heuristically the subset of genera in the phylogeny that maximized the PD metric ( $\left.\mathrm{PD}_{\max }\right)$ for a sample size $S=10,20,30$, $40,50,60,70,80$, and $90 \%$ of the total pool $(n=13489)$, respectively. Because there are multiple subsets of size $S$ that maximize PD in a phylogeny, we produced $10 \mathrm{PD}_{\max }$ subsets of genera per alternative phylogenetic hypothesis $(\mathrm{n}=100)$ and sample size $S$. Thus, we obtained 1000 different $\mathrm{PD}_{\max }$ subsets for each sample size $S^{9}$.

Assessing the performance of the $\mathbf{P D}_{\max }$ strategy. With regard to human benefits provided by plant biodiversity, the $\mathrm{PD}_{\max }$ strategy could be considered more powerful than random selection of taxa if the former captures (i) a greater richness of plant-use categories, (ii) a greater number of plant-use records (in total and per category), and (iii) a greater equitability in the distribution of the records among the categories (Pielou's evenness index ${ }^{41}$ ). Thus, for each sample size $S$, we computed these variables using $1000 \mathrm{PD}_{\max }$ subsets and averaged the results to obtain one observed value per sample size and variable ${ }^{9}$. We used standardized effect sizes (SES) to compare observed values against null distributions generated by randomly picking subsets of $S$ taxa 1000 times:

$\mathrm{SES}=\frac{M_{\text {obs }}-M_{\text {null }}}{S D_{\text {null }}}$

where SES is the standardized effect size score for a given variable and sample size,

$\mathrm{M}_{\mathrm{obs}}$ is the observed averaged value of the variable when taxa selection is phylogenetically informed (i.e. using $\mathrm{PD}_{\max }$ subsets), $\mathrm{M}_{\text {null }}$ is the mean of the null distribution (averaged value of the variable when taxa are picked at random), and $\mathrm{SD}_{\text {null }}$ is the standard deviation of the null distribution. 
Phylogenetic diversity of plant-use categories. We computed the amount of evolutionary history (PD) that is encapsulated in each plant-use category in our dataset ${ }^{4}$. PD is not statistically independent of taxa richness, which differed very greatly between the categories (Supplementary Table S2). Therefore, in order to make PD values comparable between them, we computed SES scores using equation 1. Null distributions of PD were generated for each category by shuffling taxa labels across the phylogenetic tips 1000 times $^{42}$, and SES scores were averaged across the 100 phylogenetic hypotheses. All analyses were conducted in $\mathrm{R}^{43}$ using the packages picante ${ }^{44}$, phytools $^{38}$ and the greedyPD function developed by Mazel et al. ${ }^{9}$.

Continental-scale analyses. In order to assess whether the relationship between PD and plant benefits holds across floras that have evolved in distinct biogeographic regions, we also conducted all the analyses described above at the continental scale. To do so, we compiled a checklist of the native genera of each TDWG level-1 region (Biodiversity Information Standards $\left.{ }^{45}\right)$, namely, Africa $(\mathrm{n}=4487)$, Australasia $(\mathrm{n}=2067)$, Europe + Asia-Temperate $(\mathrm{n}=$ 4117), North America $(\mathrm{n}=3307)$, Asia-Tropical $(\mathrm{n}=4071)$ and South America $(\mathrm{n}=4783)$, using distributional information available in Plants of the World Online $e^{32}$ and also Mabberley's plant-book $k^{15}$ in the few cases where this information could not be retrieved from the former source. The TDWG regions "Pacific" (minor Pacific islands) and "Antarctic" were disregarded because they showed comparatively lower diversities, and "Europe" and "Asia-Temperate" were merged into one single unit because the taxonomic turnover between the two regions (i.e. $\beta_{\text {sim }}$ distance ${ }^{46}$ ) was very low (Table S5), meaning that most of the genus-level flora of "Europe" (the less diverse of the two) is shared with that of "Asia-Temperate". Thus, we finally analyzed six continental datasets separately. We note that widespread genera might not always include useful species across their entire distribution range, which would lead to overestimating the ethnofloras of the regions. Thus, in order to account for this potential bias, we also conducted the continental-scale analyses using only the genera that were endemic to each region (Africa $=$ 2294; Australasia $=776$; Europe + Asia-Temperate $=1887$; North America $=824$; AsiaTropical $=809 ;$ South America $=2387$. 


\section{Data availability}

The plant-use dataset will be immediately published as an Open Access data paper upon acceptance of this article. All the information included in the dataset was obtained from the fourth edition of Mabberley's plant-book.

\section{Code availability}

All the code used in this research is available as functions that were either implemented in published R packages or provided as supplementary material in a previous Open Access study.

\section{References}

1. D. P. Faith, S. Magallón, A. P. Hendry, E. Conti, T. Yahara, M. J. Donoghue, Evosystem services: an evolutionary perspective on the links between biodiversity and human well-being. Curr. Opin. Env. Sust. 2, 66-74 (2010).

2. R. Cámara-Leret, S. Faurby, M. J. Macía, H. Balslev, B. Göldel, J.-C. Svenning, W. D. Kissling, N. Rønsted, C. H. Saslis-Lagoudakis, Fundamental species traits explain provisioning services of tropical American palms. Nat Plants. 3, 16220 (2017).

3. C. Oka, M. Aiba, T. Nakashizuka, Phylogenetic clustering in beneficial attributes of tree species directly linked to provisioning, regulating and cultural ecosystem services. Ecol. Indic. 96, 477-495 (2019).

4. D. P. Faith, Conservation evaluation and phylogenetic diversity. Biol. Conserv. 61, 110 (1992).

5. R. I. Vane-Wright, C. J. Humphries, P. H. Williams, What to protect?-Systematics and the agony of choice. Biol. Conserv. 55, 235-254 (1991).

6. R. H. Crozier, Genetic diversity and the agony of choice. Biol. Conserv. 61, 11-15 (1992).

7. C. M. Tucker, T. Aze, M. W. Cadotte, J. L. Cantalapiedra, C. Chisholm, S. Díaz, R. Grenyer, D. Huang, F. Mazel, W. D. Pearse, Assessing the utility of conserving evolutionary history. Biol. Rev. (2019).

8. N. R. Owen, R. Gumbs, C. L. Gray, D. P. Faith, Global conservation of phylogenetic diversity captures more than just functional diversity. Nat. Commun. 10, 859 (2019).

9. F. Mazel, M. W. Pennell, M. W. Cadotte, S. Diaz, G. V. Dalla Riva, R. Grenyer, F. Leprieur, A. O. Mooers, D. Mouillot, C. M. Tucker, W. D. Pearse, Prioritizing 
phylogenetic diversity captures functional diversity unreliably. Nat. Commun. 9 (2018), doi:10.1038/s41467-018-05126-3.

10. F. Mazel, M. W. Pennell, M. W. Cadotte, S. Diaz, G. V. D. Riva, R. Grenyer, F. Leprieur, A. O. Mooers, D. Mouillot, C. M. Tucker, W. D. Pearse, Reply to: “Global conservation of phylogenetic diversity captures more than just functional diversity." Nat. Commun. 10, 858 (2019).

11. F. Forest, R. Grenyer, M. Rouget, T. J. Davies, R. M. Cowling, D. P. Faith, A. Balmford, J. C. Manning, Ş. Procheş, M. van der Bank, others, Preserving the evolutionary potential of floras in biodiversity hotspots. Nature. 445, 757-760 (2007).

12. F. E. M. Cook, Economic Botany Data Collection Standard. International Working Group on Taxonomic Databases for Plant Sciences (TDWG). Royal Botanic Gardens, Kew, UK (1995).

13. S. A. Smith, J. W. Brown, Constructing a broadly inclusive seed plant phylogeny. Am. J. Bot. 105, 302-314.

14. Y. Jin, H. Qian, V.PhyloMaker: an R package that can generate very large phylogenies for vascular plants. Ecography. 42, 1353-1359 (2019).

15. D. J. Mabberley, Mabberley's plant-book: a portable dictionary of plants, their classification and uses. Fourth edition. Cambridge University Press (2017).

16. P. A. Cox, Will tribal knowledge survive the millennium? Science. 287, 44-45 (2000).

17. R. Cámara-Leret, N. Paniagua-Zambrana, H. Balslev, M. J. Macía, Ethnobotanical knowledge is vastly under-documented in northwestern South America. PLOS ONE. 9, e85794 (2014).

18. R. Cámara-Leret, Z. Dennehy, Information gaps in indigenous and local knowledge for science-policy assessments. Nat. Sustain. 2, 736-741 (2019).

19. V. Novotny, Y. Basset, S. E. Miller, G. D. Weiblen, B. Bremer, L. Cizek, P. Drozd, Low host specificity of herbivorous insects in a tropical forest. Nature. 416, 841-844 (2002).

20. G. S. Gilbert, R. Magarey, K. Suiter, C. O. Webb, Evolutionary tools for phytosanitary risk analysis: phylogenetic signal as a predictor of host range of plant pests and pathogens. Evol. Appl. 5, 869-878 (2012).

21. J. Calatayud, J. L. Hórreo, J. Madrigal-González, A. Migeon, M. Á. Rodríguez, S. Magalhães, J. Hortal, Geography and major host evolutionary transitions shape the resource use of plant parasites. PNAS. 113, 9840-9845 (2016).

22. G. T. Pecl, M. B. Araújo, J. D. Bell, J. Blanchard, T. C. Bonebrake, I.-C. Chen, T. D. Clark, R. K. Colwell, F. Danielsen, B. Evengård, L. Falconi, S. Ferrier, S. Frusher, R. A. Garcia, R. B. Griffis, A. J. Hobday, C. Janion-Scheepers, M. A. Jarzyna, S. Jennings, J. Lenoir, H. I. Linnetved, V. Y. Martin, P. C. McCormack, J. McDonald, N. 
J. Mitchell, T. Mustonen, J. M. Pandolfi, N. Pettorelli, E. Popova, S. A. Robinson, B. R. Scheffers, J. D. Shaw, C. J. B. Sorte, J. M. Strugnell, J. M. Sunday, M.-N. Tuanmu, A. Vergés, C. Villanueva, T. Wernberg, E. Wapstra, S. E. Williams, Biodiversity redistribution under climate change: Impacts on ecosystems and human well-being. Science. 355 (2017).

23. P. Lehmann, T. Ammunét, M. Barton, A. Battisti, S. D. Eigenbrode, J. U. Jepsen, G. Kalinkat, S. Neuvonen, P. Niemelä, J. S. Terblanche, B. Økland, C. Björkman, Complex responses of global insect pests to climate warming. Front. Ecol. Environ. 18, 141-150 (2020).

24. R. F. P. de Lucena, P. M. de Medeiros, E. de L. Araújo, A. G. C. Alves, U. P. de Albuquerque, The ecological apparency hypothesis and the importance of useful plants in rural communities from Northeastern Brazil: An assessment based on use value. J. Environ. Manag. 96, 106-115 (2012).

25. G. Menendez-Baceta, L. Aceituno-Mata, V. Reyes-García, J. Tardío, M. Salpeteur, M. Pardo-de-Santayana, The importance of cultural factors in the distribution of medicinal plant knowledge: A case study in four Basque regions. J. Ethnopharmacol. 161, 116-127 (2015).

26. C. O. Webb, D. D. Ackerly, M. A. McPeek, M. J. Donoghue, Phylogenies and Community Ecology. Annu. Rev. Ecol. Syst. 33, 475-505 (2002).

27. Intergovernmental Science-Policy Platform on Biodiversity and Ecosystem Services Global. Information on scoping for the thematic assessment of sustainable use of wild species. IPBES (2018). Available at: https://ipbes.net/sustainable-use-wild-species$\underline{\text { assessment }}$

28. M. Pardo-de-Santayana, M. Macía, The benefits of traditional knowledge. Nature. 518, 487-488 (2015).

29. S. Díaz, U. Pascual, M. Stenseke, B. Martín-López, R. T. Watson, Z. Molnár, R. Hill, K. M. A. Chan, I. A. Baste, K. A. Brauman, S. Polasky, A. Church, M. Lonsdale, A. Larigauderie, P. W. Leadley, A. P. E. van Oudenhoven, F. van der Plaat, M. Schröter, S. Lavorel, Y. Aumeeruddy-Thomas, E. Bukvareva, K. Davies, S. Demissew, G. Erpul, P. Failler, C. A. Guerra, C. L. Hewitt, H. Keune, S. Lindley, Y. Shirayama, Assessing nature's contributions to people. Science. 359, 270-272 (2018).

30. A. Antonelli, et al. (205 authors), State of the world's plants and fungi 2020. Royal Botanic Gardens, Kew (2020).

31. T. Ulian, M. Diazgranados, S. Pironon, S. Padulosi, U. Liu, L. Davies, M.-J. R. Howes, J. S. Borrell, I. Ondo, O. A. Pérez-Escobar, S. Sharrock, P. Ryan, D. Hunter, M. A. Lee, C. Barstow, Ł. Łuczaj, A. Pieroni, R. Cámara-Leret, A. Noorani, C. Mba, R. N. Womdim, H. Muminjanov, A. Antonelli, H. W. Pritchard, E. Mattana, 
Unlocking plant resources to support food security and promote sustainable agriculture. Plants, People, Planet. 2, 421-445 (2020).

32. Plants of the World Online. Facilitated by the Royal Botanic Gardens, Kew. Published on the Internet; http://www.plantsoftheworldonline.org/

33. A. E. Zanne, D. C. Tank, W. K. Cornwell, J. M. Eastman, S. A. Smith, R. G. FitzJohn, D. J. McGlinn, B. C. O’Meara, A. T. Moles, P. B. Reich, D. L. Royer, D. E. Soltis, P. F. Stevens, M. Westoby, I. J. Wright, L. Aarssen, R. I. Bertin, A. Calaminus, R. Govaerts, F. Hemmings, M. R. Leishman, J. Oleksyn, P. S. Soltis, N. G. Swenson, L. Warman, J. M. Beaulieu, Three keys to the radiation of angiosperms into freezing environments. Nature. 506, 89-92 (2014).

34. The Plant List. Version 1.1. Published on the Internet; http://www.theplantlist.org/

35. T. F. Rangel, R. K. Colwell, G. R. Graves, K. Fučíková, C. Rahbek, J. A. F. DinizFilho, Phylogenetic uncertainty revisited: Implications for ecological analyses. Evolution. 69, 1301-1312 (2015).

36. S. Federhen, The NCBI Taxonomy database. Nucleic Acids Res. 40, D136-D143 (2012).

37. E. Hörandl, T. F. Stuessy, Paraphyletic groups as natural units of biological classification. Taxon. 59, 1641-1653 (2010).

38. L. J. Revell, phytools: an R package for phylogenetic comparative biology (and other things). Methods Ecol. Evol. 3, 217-223 (2012).

39. A. S. L. Rodrigues, K. J. Gaston, Maximising phylogenetic diversity in the selection of networks of conservation areas. Biol. Conserv. 105, 103-111 (2002).

40. M. Bordewich, A. G. Rodrigo, C. Semple, Selecting taxa to save or sequence: desirable criteria and a greedy solution. Syst. Biol. 57, 825-834 (2008).

41. E. C. Pielou, The measurement of diversity in different types of biological collections. J. Theor. Biol. 13, 131-144 (1966).

42. S. W. Kembel, Disentangling niche and neutral influences on community assembly: assessing the performance of community phylogenetic structure tests. Ecol. Lett. 12, 949-960 (2009).

43. R Core Team. R: A language and environment for statistical computing. (R foundation for statistical computing Vienna, Austria, 2020).

44. S. W. Kembel, P. D. Cowan, M. R. Helmus, W. K. Cornwell, H. Morlon, D. D. Ackerly, S. P. Blomberg, C. O. Webb, Picante: R tools for integrating phylogenies and ecology. Bioinformatics. 26, 1463-1464 (2010).

45. Brummitt, R. K. (2001). World geographical scheme for recording plant distributions (PDF) (2nd ed.). International working group on taxonomic databases for plant sciences (TDWG). 
46. A. Baselga, Partitioning the turnover and nestedness components of beta diversity. Glob. Ecol. Biogeogr. 19, 134-143 (2010).

\section{Acknowledgments}

We thank the Scientific Computation Center of Andalusia (CICA) for the computing services they provided and Herlander Lima for his assistance in downloading plant distributional information from the web. This work was supported by the Regional Government of the Community of Madrid and the University of Alcalá through the project "Plant evolutionary history and human well-being in a changing world; assessing theoretical foundations using empirical evidence and new phylogenetic tools", which was granted to RMV (CM/JIN/2019005). RMV was supported by the TALENTO program of the Regional Government of the Community of Madrid (2018-T2/AMB-10332). MAR was supported by the Ministry of Science and Innovation of Spain (grant CGL2017-86926-P).

\section{Author contributions}

RMV conceived the ideas, led the assemblage of the plant-use dataset with the help of MPS and DJM, conducted the analyses and led the writing. CR led the assemblage of the continental datasets. MAR suggested using Mabberley's plant-book as the source of global plant-use information. All the authors read, edited and commented on the manuscript.

\section{Competing interests}

The authors declare no competing interests.

\section{Additional information}

Supplementary information is available for this paper at XXX 

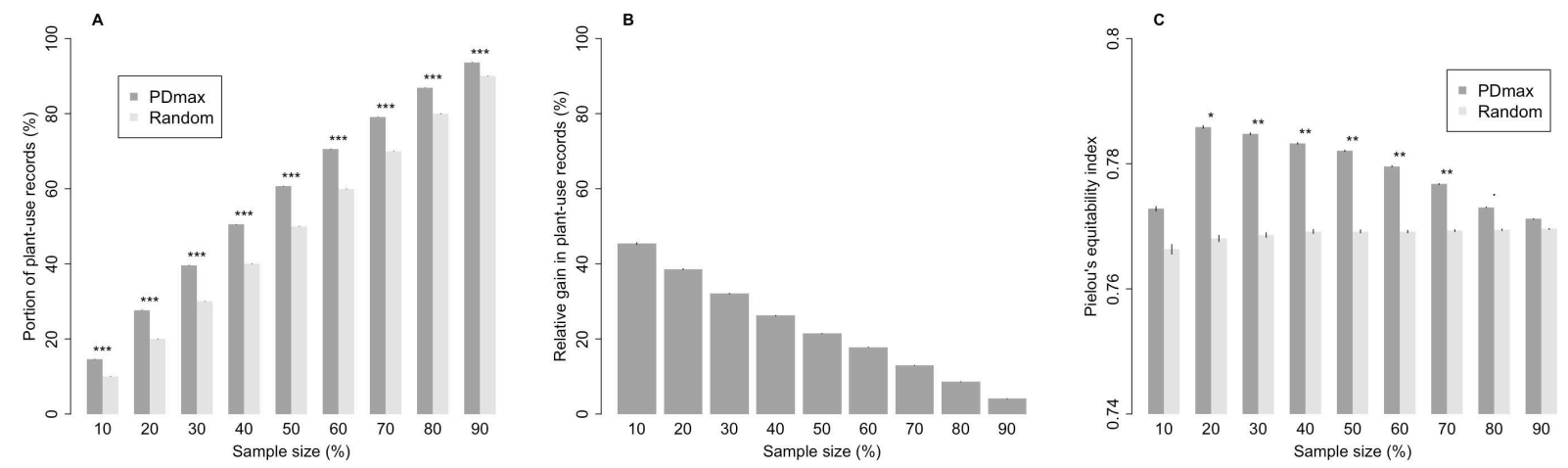

Fig. 1. Relative gain in plant-use records and equitability in their distribution among categories. a, Portion of the total pool of plant-use records $(n=9478$ counted for all use categories combined) retrieved with the $\mathrm{PD}_{\max }$ and random selection strategies across sample sizes. $\mathrm{b}$, Gain in plant-use records obtained with $\mathrm{PD}_{\max }$ relative to random selection across sample sizes. $\mathrm{c}$, 480 Equitability (Pielou's evenness index) in the distribution of plant-use records among the 28 481 categories with $\mathrm{PD}_{\max }$ and random sampling strategies across sample sizes. The symbols in a and c indicate statistical significance (based on SES scores) for a nominal alpha of $10 \%$ “.”, $5 \%$ " “*”, $1 \%$ “**” and $0.1 \%$ “***”, respectively (two-tailed tests), and the vertical thin bars at the center of the percentage bars represent confidence intervals at $95 \%$. 

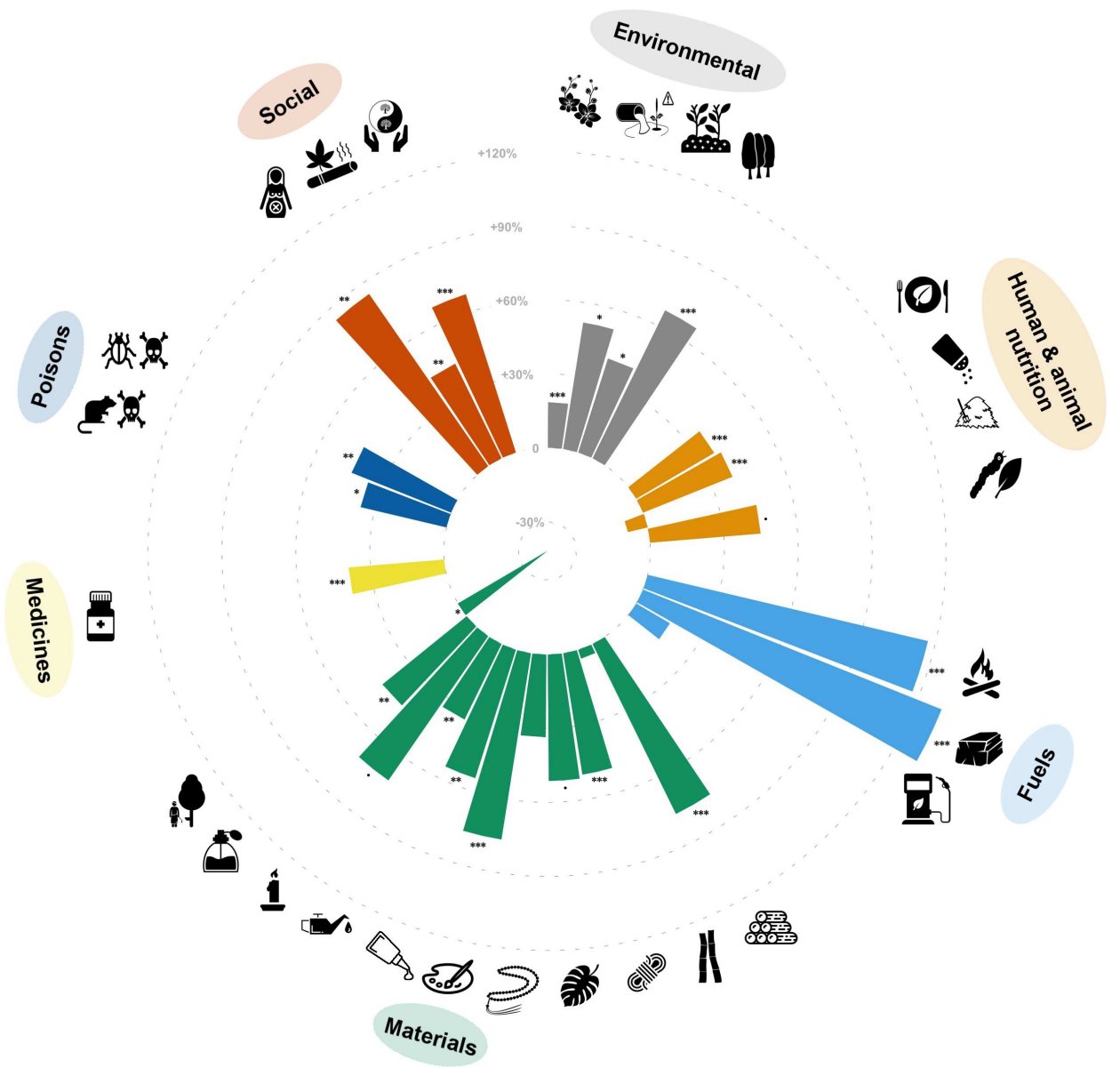

Fig. 2. Relative gains in plant-use records per category. The bars represent the relative gains obtained with $\mathrm{PD}_{\max }$ relative to random selection at $\mathrm{S}=20 \%$ of the total pool of taxa, the sample size at which the maximum equitability in the distribution of records among use categories was observed (see Fig. 1c). The symbols on the bars indicate statistical significance (based on SES scores) for a nominal alpha of 10\% “"”, 5\% “*”, $1 \%$ “**” and $0.1 \%$ “***”, respectively (twotailed tests). The colours represent different groups of categories following the Economic Botany Data Collection Standard (see Supplementary Table S1). Note that in order to optimize the visibility of the figure, values below the $-30 \%$ threshold do not scale linearly (only one category with relative gain at $-61 \%$ ). From twelve o'clock and clockwise: (1) ornamental, (2) bioindicators and bioremediators, (3) soil improvers, (4) hedges and shelters, (5) human food, (6) human food additives, (7) vertebrate food, (8) invertebrate food, (9) fuelwood, (10) charcoal, (11) biofuels, (12) timber, (13) stems, (14) fibres, (15) leaves, (16) seeds and fruits, (17) tannins and dyestuffs, (18) resins and gums, (19) lipids, (20) waxes, (21) scents, (22) rubber, (23) medicines, (24) vertebrate poisons, (25) invertebrate poisons, (26) antifertility agents, (27) smoking materials and drugs, (28) symbolism, magic and inspiration. 

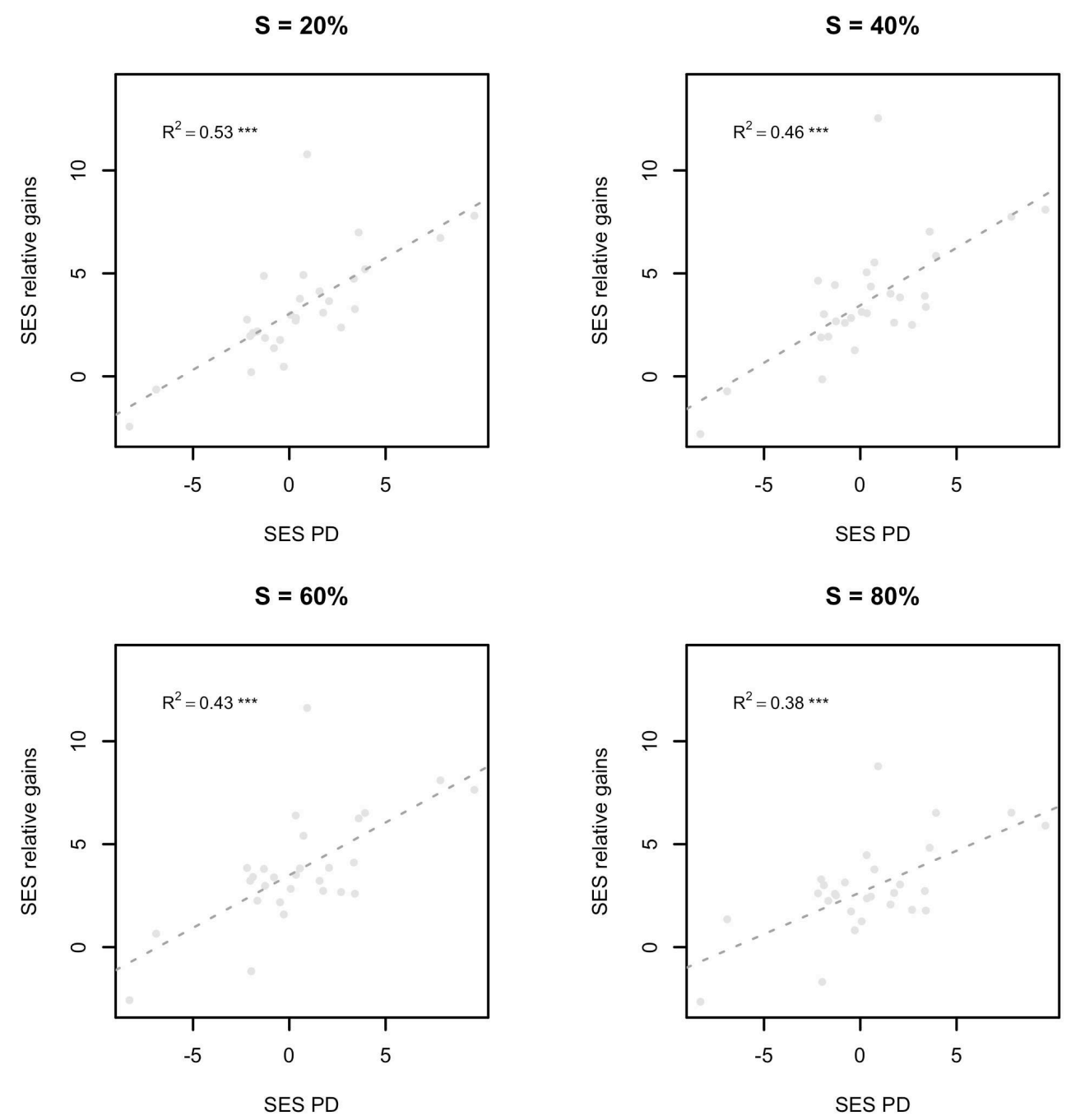

SES PD

SES PD

Fig. 3. Relationship between the phylogenetic structure of plant-use categories and relative gains per category under the $\mathbf{P D}_{\max }$ strategy. The dotted lines represent the regression models between the phylogenetic structure of plant-use categories (SES scores of PD averaged across 100 phylogenetic hypotheses) and SES scores of the relative gains per category across different sample sizes ( $\mathrm{S}=20,40,60$ and $80 \%$ of the total pool). All regressions were significant for a nominal alpha of $0.1 \%$. 


\section{Figures}
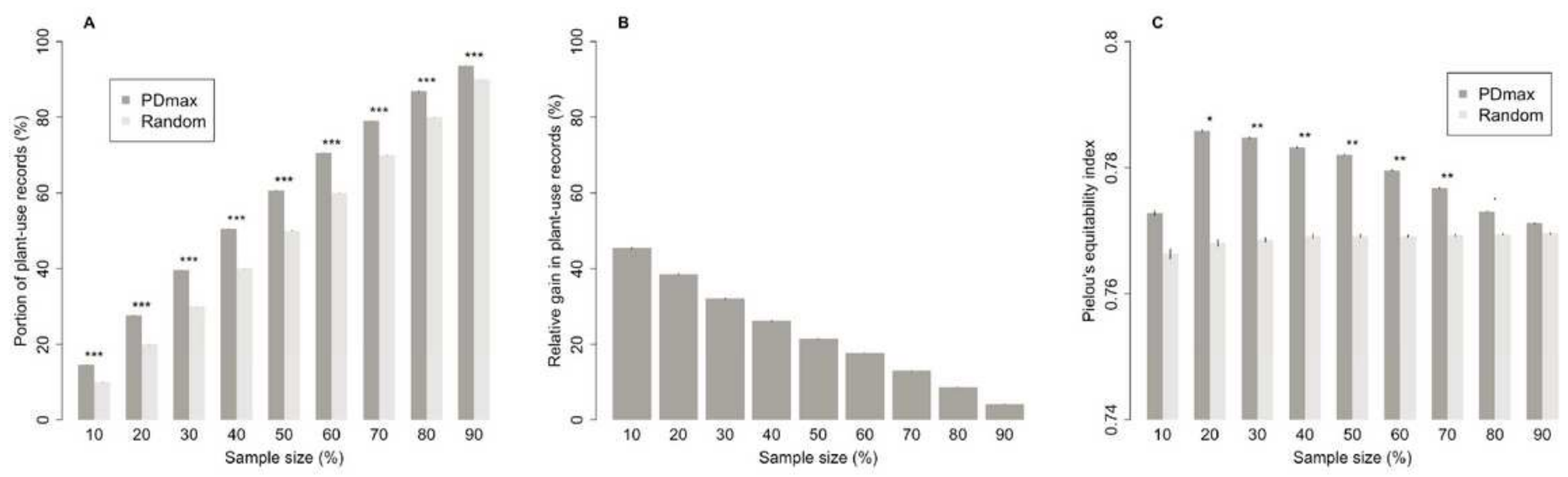

\section{Figure 1}

Relative gain in plant-use records and equitability in their distribution among categories. a, Portion of the total pool of plant-use records ( $n=9478$ counted for all use categories combined) retrieved with the PDmax and random selection strategies across sample sizes. b, Gain in plant-use records obtained with PDmax relative to random selection across sample sizes. c, Equitability (Pielou's evenness index) in the distribution of plant-use records among the 28 categories with PDmax and random sampling strategies across sample sizes. The symbols in a and c indicate statistical significance (based on SES scores) for a

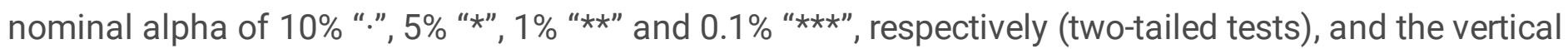
thin bars at the center of the percentage bars represent confidence intervals at $95 \%$. 


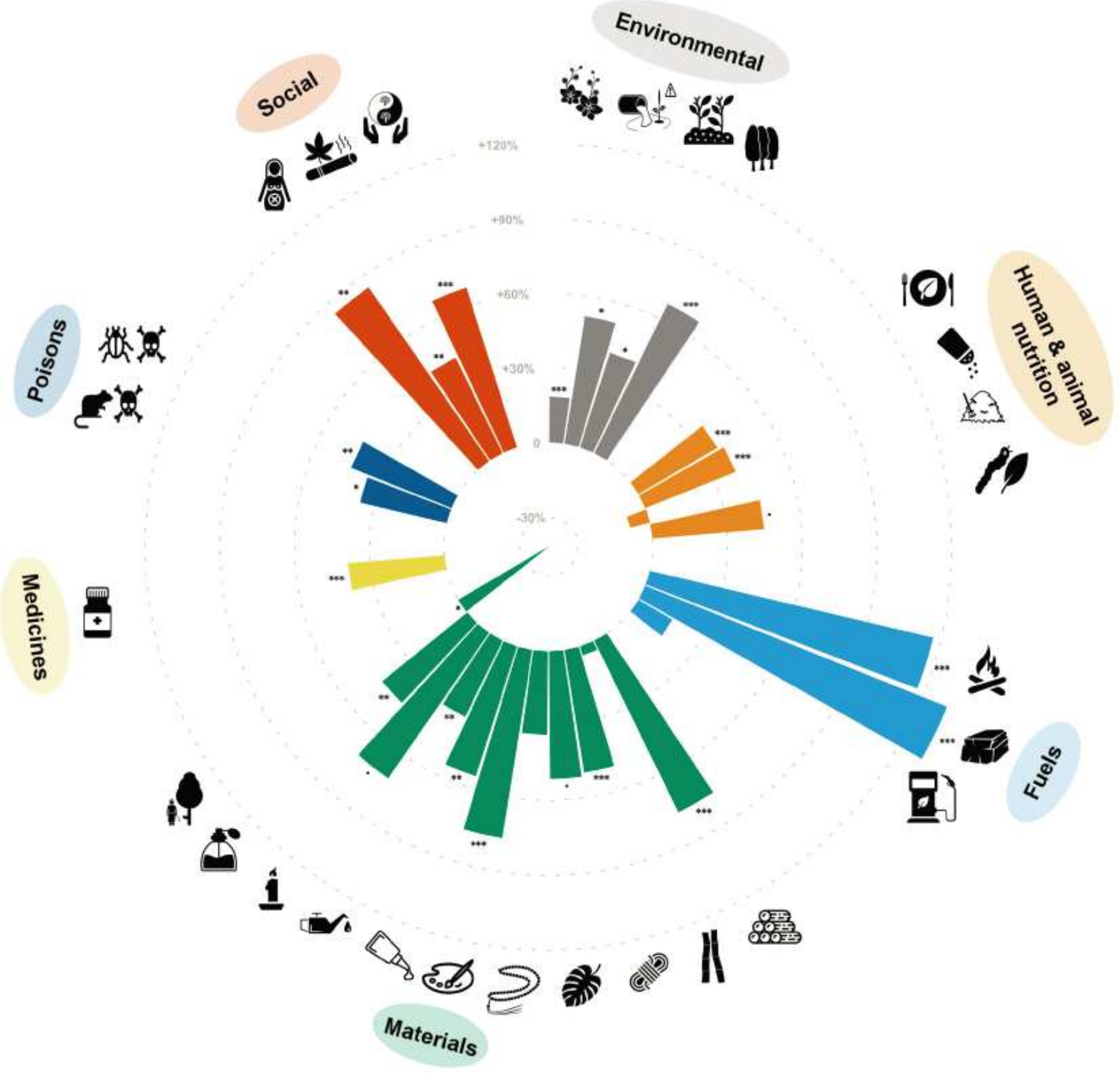

Figure 2

Relative gains in plant-use records per category. The bars represent the relative gains obtained with PDmax relative to random selection at $S=20 \%$ of the total pool of taxa, the sample size at which the maximum equitability in the distribution of records among use categories was observed (see Fig. 1c). The symbols on the bars indicate statistical significance (based on SES scores) for a nominal alpha of $10 \%$

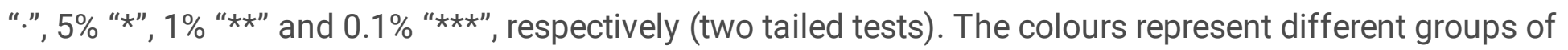
categories following the Economic Botany Data Collection Standard (see Supplementary Table S1). Note that in order to optimize the visibility of the figure, values below the $-30 \%$ threshold do not scale linearly (only one category with relative gain at $-61 \%$ ). From twelve o'clock and clockwise: (1) ornamental, (2) 
bioindicators and bioremediators, (3) soil improvers, (4) hedges and shelters, (5) human food, (6) human food additives, (7) vertebrate food, (8) invertebrate food, (9) fuelwood, (10) charcoal, (11) biofuels, (12) timber, (13) stems, (14) fibres, (15) leaves, (16) seeds and fruits, (17) tannins and dyestuffs, (18) resins and gums, (19) lipids, (20) waxes, (21) scents, (22) rubber, (23) medicines, (24) vertebrate poisons, (25) invertebrate poisons, (26) antifertility agents, (27) smoking materials and drugs, (28) symbolism, magic and inspiration.

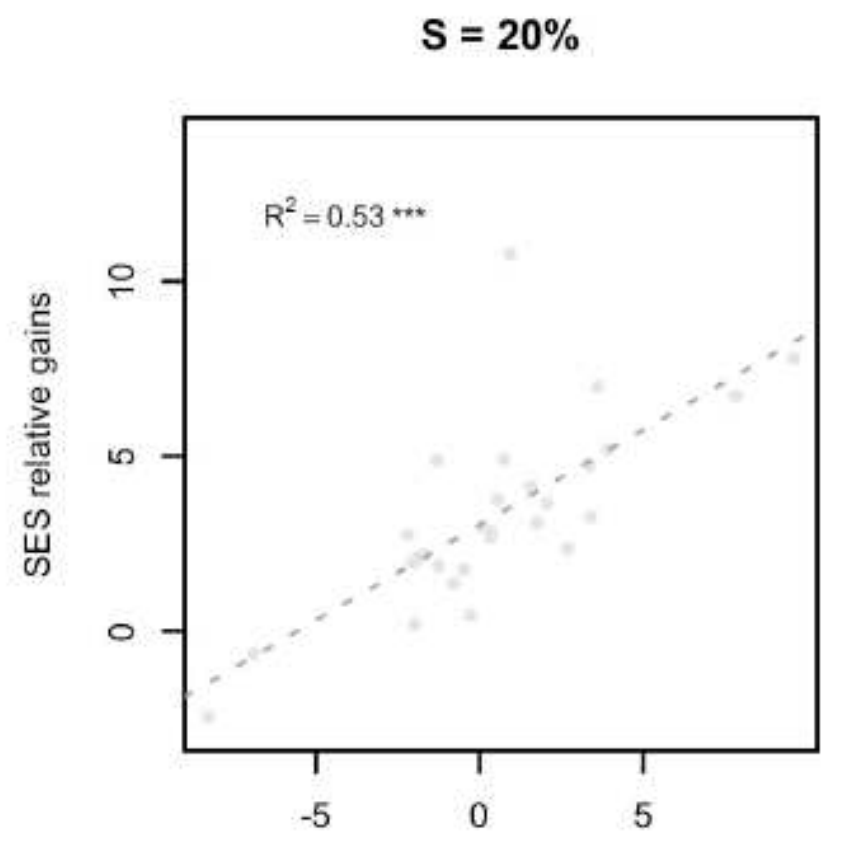

SES PD

$$
S=60 \%
$$

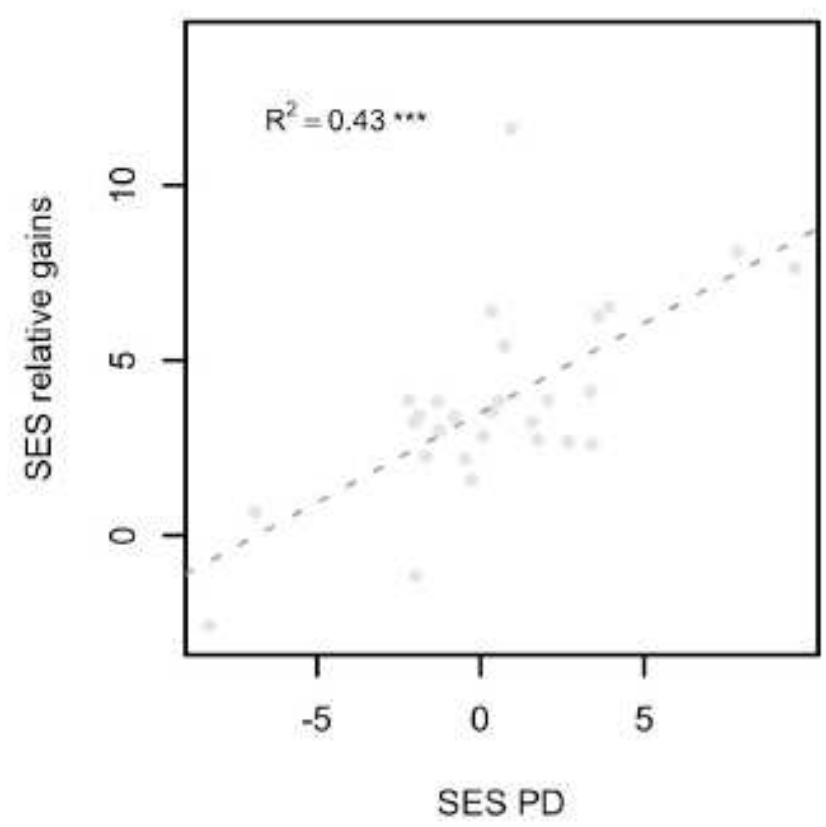

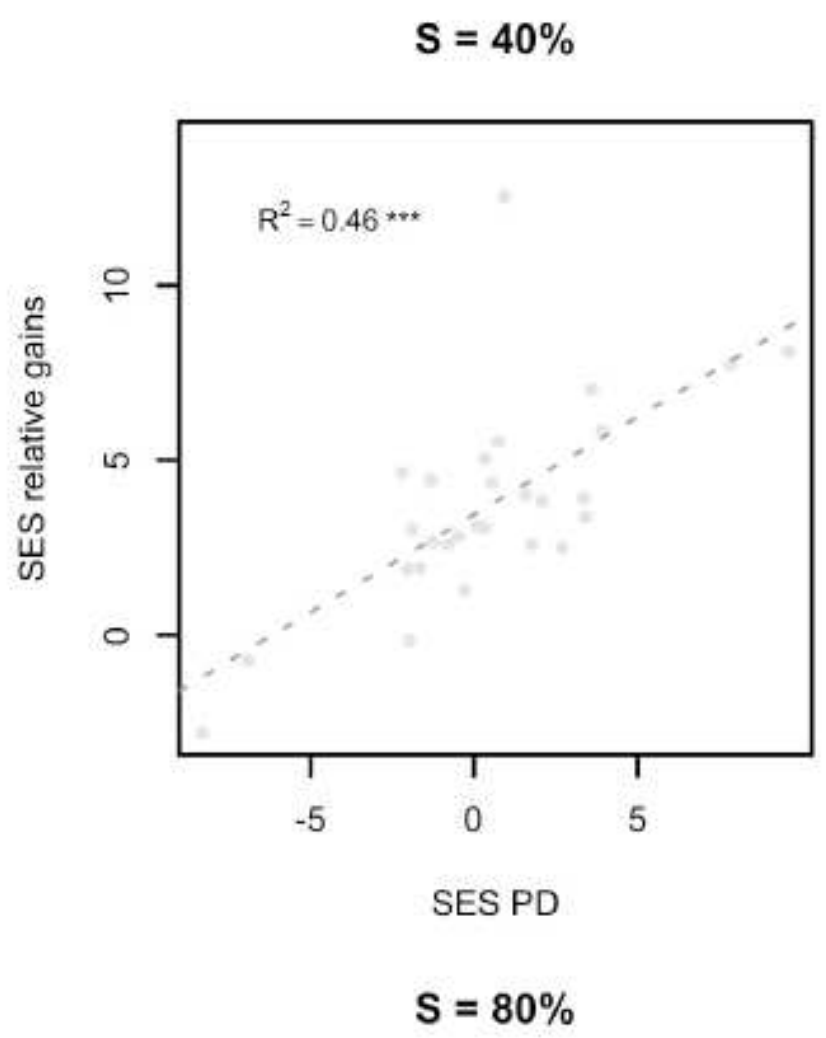

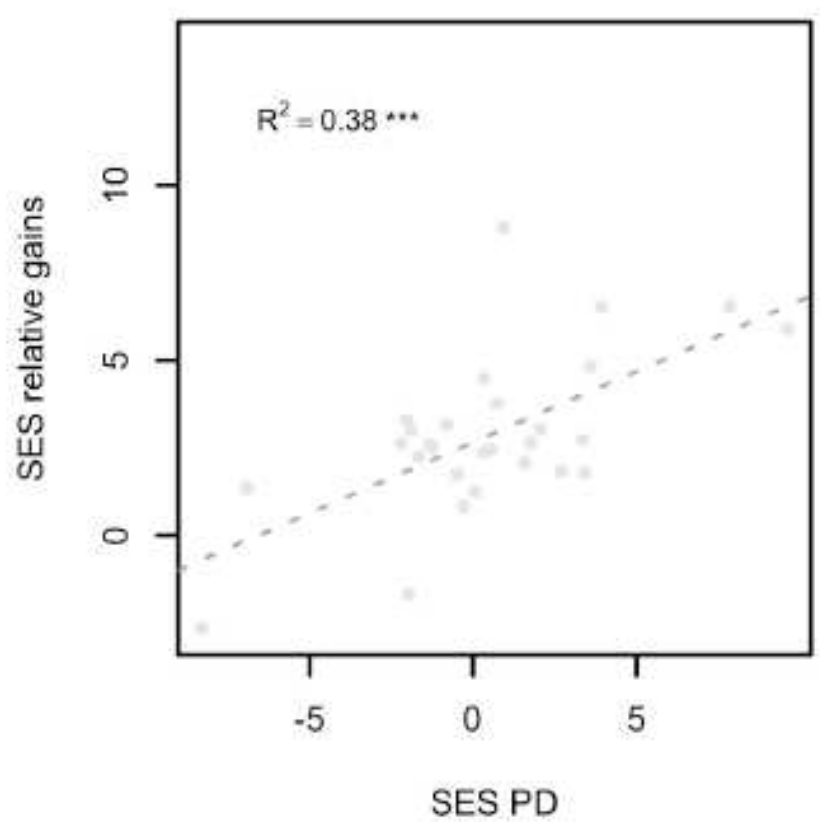

Figure 3 
Relationship between the phylogenetic structure of plant-use categories and relative gains per category under the PDmax strategy. The dotted lines represent the regression models between the phylogenetic structure of plant-use categories (SES scores of PD averaged across 100 phylogenetic hypotheses) and SES scores of the relative gains per category across different sample sizes $(S=20,40,60$ and $80 \%$ of the total pool). All regressions were significant for a nominal alpha of $0.1 \%$.

\section{Supplementary Files}

This is a list of supplementary files associated with this preprint. Click to download.

- SupplementaryNEE.pdf

- TableS3.xIsx

- TableS4.xlsx 